Open Access

\title{
Evaluation of Potential Reference Genes for Quantitative RT-PCR Analysis in Fusarium graminearum under Different Culture Conditions
}

\author{
Hee-Kyoung Kim and Sung-Hwan Yun* \\ Department of Medical Biotehcnology, Soonchunhyang University, Asan 336-745, Korea \\ (Received on October 25, 2011; Accepted on November 2, 2011)
}

The filamentous fungus Fusarium graminearum is an important cereal pathogen. Although quantitative realtime PCR (qRT-PCR) is commonly used to analyze the expression of important fungal genes, no detailed validation of reference genes for the normalization of qRT-PCR data has been performed in this fungus. Here, we evaluated 15 candidate genes as references, including those previously described as housekeeping genes and those selected from the whole transcriptome sequencing data. By a combination of three statistical algorithms (BestKeeper, geNorm, and NormFinder), the variation in the expression of these genes was assessed under different culture conditions that favored mycelial growth, sexual development, and trichothecene mycotoxin production. When favoring mycelial growth, $G z F L O$ and $G z U B H$ expression were most stable in complete medium. Both $E F 1 A$ and $G z R P S 16$ expression were relatively stable under all conditions on carrot agar, including mycelial growth and the subsequent perithecial induction stage. These two genes were also most stable during trichothecene production. For the combined data set, $G z U B H$ and $E F 1 A$ were selected as the most stable. Thus, these genes are suitable reference genes for accurate normalization of qRT-PCR data for gene expression analyses of $\boldsymbol{F}$. graminearum and other related fungi.

Keywords : Fusarium graminearum, gene expression, quantitative real-time PCR, reference genes

Quantitative real-time PCR (qRT-PCR) is the most sensitive and specific method for quantifying mRNA expression levels of individual target genes of interest. qRT-PCR expression analysis has several advantages over other conventional methods, but requires data normalization with appropriate reference gene(s) whose expression should be unaffected throughout the study conditions (Anderson et al., 2004; Bustin et al., 2004; Gutierrez et al., 2008). Several housekeeping genes involved in basic cellular functions,

\footnotetext{
*Corresponding author.

Phone) +82-41-530-1288, FAX) +82-41-530-3085

E-mail)sy14@sch.ac.kr
}

such as ribosomal genes, and those encoding actin, betatubulin (BTUB), translation elongation factor (EF1), and glyceraldehyde-3-phosphate dehydrogenase (GAPDH) have been used as reference genes for qRT-PCR in humans and other model organisms. However, several studies have demonstrated that some of these traditional housekeeping genes are unsuitable references because their expression levels vary under certain conditions (Czechowski et al., 2005; Dheda et al., 2004; Lee et al., 2002; Suzuki et al., 2000). Therefore, it is necessary to evaluate candidate reference genes for qRT-PCR expression analysis under specific experimental conditions in the organism to be studied.

The filamentous fungus Fusarium graminearum (telomorph: Gibberella zeae) is an important cereal pathogen, and produces mycotoxins that are harmful to humans and animals (Desjardins and Proctor, 2007; McMullen et al., 1997). Complete genome sequencing of $F$. graminearum (Cuomo et al., 2007) has allowed for genome-wide expression profiling using DNA microarrays under various developmental and physiological conditions, such as mycelial growth (Güldener et al., 2006) sexual development (Hallen et al., 2007), germination (Seong et al., 2008) and pathogenesis (Güldener et al., 2006; Stephens et al., 2008). Although the microarray data in these studies were not validated by qRT-PCR, it has become the most common method to obtain specific expression patterns of genes identified from gene expression profiles (Chen et al., 2011; Liu et al., 2011; Pandolfi et al., 2010) or functional studies (Lee et al., 2009; Lysøe et al., 2009). However, the expression stabilities of the housekeeping genes used as references for qRT-PCR analysis in even these studies have not been properly validated.

The objective of this study was to identify $F$. graminearum reference genes suitable for normalization of qRTPCR data under different conditions such as mycelial growth, sexual development, and mycotoxin production. We selected 15 candidate references, including the common housekeeping genes used in previous studies (Chen et al., 2011; Lee et al., 2009; Liu et al., 2011; Lysøe et al., 2009; Pandolfi et al., 2010) and a new set of genes selected from 
the whole transcriptome sequencing (RNA-seq) analysis of a F. graminearum strain (unpublished results). By comparing the variation in the expression of these genes under various conditions using three different algorithms [geNorm (Vandesompele et al., 2002), NormFinder (Anderson et al., 2004), and BestKeeper (Pfaffl et al., 2004)] we identified suitable reference genes that can be used in subsequent expression studies.

\section{Materials and Methods}

Fungal strain and growth conditions. The $F$. graminearum $\mathrm{PH}-1$ strain used in this study belongs to lineage 7 of the $F$. graminearum species complex (O'Donnell et al., 2000), and its genome has been sequenced (http:// www.broadinstitute.org/annotation/genome/

fusarium graminearum/MultiHome.html). It was retrieved from $25 \%$ glycerol stock cultures stored at $-80{ }^{\circ} \mathrm{C}$ and maintained on potato dextrose agar (PDA; Difco Laboratories, USA). We investigated four different culture conditions. Fungal growth in complete medium (CM) liquid culture, performed as previously described (Leslie and Summerell, 2006), represents mycelial (vegetative) growth under nutrient-rich conditions. Carrot agar (Leslie and Summerell, 2006) represents sexual development in $F$. graminearum, and favors two different developmental stages. The first 6-day growth on carrot agar represents the formation of aerial mycelia under nitrogen starvation conditions, which is a prerequisite for sexual development. The subsequent 6-day growth on the same medium, as previously described (Lee et al., 2006), represents the formation of sexual fruiting bodies (perithecia). Mycelial growth in defined liquid medium amended with agmatine (AG), performed as previously described (Gardiner et al., 2009), represents trichothecene production; toxin accumulation peaks at days 6-7 (Gardiner et al., 2009). The time points for RNA extraction under each growth condition were as follows: days 2, 4, and 6 in CM; days 2, 4, 6 on carrot agar for aerial mycelia; days 2, 4, 6 on carrot agar after removing aerial mycelia grown for 6 days on carrot agar; days 3 and 6 in AG liquid medium.

RNA extraction. The fungal mycelia or perithecia were harvested from each culture at the chosen time points and ground to fine powder in liquid nitrogen (Lee et al., 2006). Total RNA from the samples was extracted using the Total RNA extraction kit (iNtRON Biotechnolgy, Korea) according to the manufacturer's protocol. All RNA samples were assessed for quality by agarose gel electrophoresis and quantified using ultraviolet spectrophotometry at a $260 \mathrm{~nm} /$ $280 \mathrm{~nm}$ absorbance ratio (between 1.8 and 2.0). The firststrand cDNA was synthesized from total RNA using
PrimeScript RT reagent kit (TaKaRa, Japan).

Reference genes, primer design, and PCR efficiency test. Fifteen candidate reference genes were selected (Table 1). Seven were $F$. graminearum orthologs of common housekeeping genes that are frequently used as references for data normalization. The remaining eight were selected from the F. graminearum PH-1 genome based on the read frequencies of individual mRNAs by RNA-seq analysis, each of which was expressed constitutively at similar levels under the different conditions described above (unpublished results). Primers for the newly selected candidate genes were designed using PrimerSelect program (DNASTAR, USA), and those for the commonly used housekeeping genes were obtained directly from the published literature (Table 1). The amplification efficiencies of all genes were determined using RNA samples from a single time point in each condition followed by analysis of the slope of a serial 10-fold cDNA dilution curve (Pfaffl et al., 2001) (Table 1).

Quantitative real-time PCR and data analysis. qRT-PCR was performed with the SYBR Green Super Mix (Bio-Rad, USA) and a real-time PCR system (Eppendorf Mastercycler ep realplex4, Germany). The changes in fluorescence of the SYBR Green dye in each cycle were monitored by the system software, and the threshold cycle $(\mathrm{Ct})$ above the background for each reaction was calculated. The expression stabilities of the candidate reference genes were evaluated using three Microsoft Excel-based statistical tools, geNorm (Vandesompele et al., 2002), NormFinder (Anderson et al., 2004) and BestKeeper (Pfaffl et al., 2004). Gene expression was measured in two separate samples from each time point in each condition (biological replicates). Each individual reaction was run in triplicate (technical replicates). The average $\mathrm{Ct}$ value of each triplicate reaction was converted into linear expression values for subsequent analysis with geNorm and NormFinder.

\section{Results}

Expression analysis of candidate reference genes. qRTPCR primers generated amplicons ranging from 91 to 250 bp (Table 1). The average PCR efficiency of the primer sets (except $B T U B$ ) ranged from 1.95 to 1.99 . Although the standard deviations for three genes $(E F 1 B, U B C$, and $C Y P 2)$ were slightly higher than 0.05 , all of the average $\mathrm{E}$ values indicated that the amplification efficiencies were near the theoretical optimum level of 2 . We also observed a single peak in real-time melt-curve analysis of all the genes (data not shown). Thus, gene-specific amplification by all primers was confirmed. In all subsequent data, $B T U B$ was not considered reliable because it displayed an unexpect- 
Table 1. Selected candidate reference genes evaluated in this study

\begin{tabular}{|c|c|c|c|c|c|c|}
\hline Gene & Gene description & Primer sequence ${ }^{\mathrm{b}}\left(5^{\prime}-3^{\prime}\right)$ & $\begin{array}{l}\text { Amplicon } \\
\text { size (bp) }\end{array}$ & $\begin{array}{l}\mathrm{Tm} \\
\left({ }^{\circ} \mathrm{C}\right)\end{array}$ & $\mathrm{E}^{\mathrm{c}}$ & Reference \\
\hline \multirow[t]{2}{*}{$E F 1 B$} & \multirow[t]{2}{*}{$\begin{array}{r}\text { FGSG_01008.3 eukaryotic translation } \\
\text { elongation factor } 1 \text { beta }\end{array}$} & $\begin{array}{l}\text { GAGTACCGCAAGAAGAAGGAGAA- } \\
\text { CAAG/ }\end{array}$ & 176 & 60.8 & \multirow{2}{*}{\multicolumn{2}{|c|}{$\begin{array}{c}1.95 \pm 0.06 \text { Lee et al. } \\
2009\end{array}$}} \\
\hline & & ACCAAAACCAACGGGGACGAG/ & & 61.6 & & \\
\hline GAPDH & $\begin{array}{c}\text { FGSG_06257.3 glyceraldehyde-3-phos- } \\
\text { phate dehydrogenase }\end{array}$ & $\begin{array}{l}\text { ACCACCGTCCACTCCTACAC/ } \\
\text { GGCGAACAGTCAAGTCAACA }\end{array}$ & 219 & $\begin{array}{l}60.0 \\
70.0\end{array}$ & \multicolumn{2}{|c|}{$\begin{array}{r}1.97 \pm 0.02 \text { Pandolfi et } \\
\text { al., } 2010\end{array}$} \\
\hline$U B C$ & $\begin{array}{c}\text { FGSG_10805.3 ubiquitin conjugating } \\
\text { enzyme }\end{array}$ & $\begin{array}{l}\text { TCCCCTTACTCTGGCGGTGTC/ } \\
\text { TTGGGGTGGTAGATGCGTGTAGT }\end{array}$ & 103 & $\begin{array}{l}59.8 \\
59.2\end{array}$ & \multicolumn{2}{|c|}{$\begin{array}{r}1.97 \pm 0.05 \text { Lysøe et } \\
\text { al., } 2009\end{array}$} \\
\hline$E F 1 A$ & $\begin{array}{r}\text { FGSG_08811.3 eukaryotic translation } \\
\text { elongation factor } 1 \text { alpha }\end{array}$ & $\begin{array}{l}\text { GGCTTTCACCGACTACCCTCCTCT/ } \\
\text { aACTTCTCGACGGCCTTGATGACAC }\end{array}$ & 91 & $\begin{array}{l}61.7 \\
62.3\end{array}$ & \multicolumn{2}{|c|}{$\begin{array}{r}1.94 \pm 0.01 \text { Lysøe et } \\
\text { al., } 2009\end{array}$} \\
\hline$B T U B$ & FGSG_09530.3 beta-tubulin & $\begin{array}{l}\text { GGTCTCGACAGCAATGGTGTT/ } \\
\text { GCTTGTGTTTTTCGTGGCAGT }\end{array}$ & 110 & $\begin{array}{l}56.1 \\
56.4\end{array}$ & \multicolumn{2}{|c|}{$\begin{array}{r}2.92 \pm 0.52 \text { Lysøe et } \\
\text { al., } 2009\end{array}$} \\
\hline CYP1 & FGSG_7439.3 cyclophilin 1 & $\begin{array}{l}\text { TCAAGCTCAAGCACACCAAGAAGG/ } \\
\text { GGTCCGCCGCTCCAGTCT }\end{array}$ & 191 & $\begin{array}{l}61.4 \\
59.6\end{array}$ & \multicolumn{2}{|l|}{$1.93 \pm 0.02$} \\
\hline CYP2 & FGSG_00777.3 cyclophilin 2 & $\begin{array}{l}\text { CTACGGTGAGAAGTTCGCTGACG/ } \\
\text { TCTTGGGCTTCTGGGTGTACTTGATGG }\end{array}$ & 250 & $\begin{array}{l}59.8 \\
65.7\end{array}$ & \multicolumn{2}{|l|}{$1.95 \pm 0.05$} \\
\hline GzHSP70 & FGSG_00838.3 heat shock protein 70 & $\begin{array}{l}\text { TCAACGGAAAGGAGCCCAACAAGT/ } \\
\text { GGGGGCGACGTCGAGGAGCAGAAT }\end{array}$ & 136 & $\begin{array}{l}63.6 \\
70.5\end{array}$ & \multicolumn{2}{|c|}{$1.95 \pm 0.04$ this study } \\
\hline$G z U B H$ & $\begin{array}{c}\text { FGSG_01231.3 ubiquitin C-teminal } \\
\text { hydrolase }\end{array}$ & $\begin{array}{l}\text { GTTCTCGAGGCCAGCAAAAAGTCA/ } \\
\text { CGAATCGCCGTTAGGGGTGTCTG }\end{array}$ & 168 & $\begin{array}{l}62.4 \\
65.2\end{array}$ & \multicolumn{2}{|c|}{$1.94 \pm 0.05$ this study } \\
\hline$G z R B P 1$ & FGSG_02497.3 RING-box protein 1 & $\begin{array}{l}\text { ATAACTGCGCCATCTGCCGTAAT/ } \\
\text { GGACAAACAGATCGAGCCTTCAAC }\end{array}$ & 165 & $\begin{array}{l}60.5 \\
59.8\end{array}$ & \multicolumn{2}{|c|}{$1.92 \pm 0.02$ this study } \\
\hline$G z F L O$ & $\begin{array}{c}\text { FGSG_05633.3 putative cell surface } \\
\text { flocculin }\end{array}$ & $\begin{array}{l}\text { GTTGAGAAGCCCGCACCTACGAC/ } \\
\text { CTCCTGGCGCTGGGGCTCCTTTGT }\end{array}$ & 179 & $\begin{array}{l}62.7 \\
70.5\end{array}$ & \multicolumn{2}{|c|}{$1.98 \pm 0.03$ this study } \\
\hline$G z D G A$ & $\begin{array}{r}\text { FGSG_06688.3 diacylglycerol } \\
\text { acyltransferase }\end{array}$ & $\begin{array}{l}\text { TGGGCCCATCTCTTCAACATTA/ } \\
\text { GCTCGCGCTCTCCTTCTACAG }\end{array}$ & 195 & $\begin{array}{l}57.9 \\
58.0\end{array}$ & \multicolumn{2}{|c|}{$1.94 \pm 0.04$ this study } \\
\hline \multicolumn{2}{|c|}{$\mathrm{Gz} C 2 \mathrm{H} 044 \mathrm{FGSG} 06701.3 \mathrm{C} 2 \mathrm{H} 2$ finger protein } & $\begin{array}{l}\text { GGCCCTTTCCCCTCTTGATGA/ } \\
\text { GCTTTTCGGCTCGGTGGTTGC }\end{array}$ & 181 & $\begin{array}{l}60.9 \\
64.5\end{array}$ & $1.99 \pm 0.03$ & 3 this study \\
\hline GzSNC1 & FGSG_08537.3 synaptobrevin & $\begin{array}{l}\text { CCGTGGGTGTGATGCGTGAC/ } \\
\text { CCTCGGCGGAAACCTTGTGCTGAA }\end{array}$ & 114 & $\begin{array}{l}61.6 \\
68.5\end{array}$ & $1.98 \pm 0.04$ & 4 this study \\
\hline GzRPS16 & $\begin{array}{l}\text { FGSG_09438.3 mitochondrial } \\
\text { ribosomal protein S16 }\end{array}$ & $\begin{array}{l}\text { GACCGATCCTTACGACGACTCTG/ } \\
\text { ATCCTTCTGCACAACACCCTTTAT }\end{array}$ & 192 & $\begin{array}{l}58.6 \\
56.8\end{array}$ & $1.99 \pm 0.01$ & this study \\
\hline
\end{tabular}

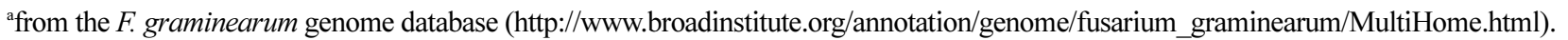

${ }^{\mathrm{b}}$ forward/reverse primer.

${ }^{c}$ average $\mathrm{E}$ value \pm standard deviation.

edly high $\mathrm{E}$ value (2.92). This may be attributable to the position of the reverse primer, spanning the third intron region in the BTUB nucleotide sequence. The $\mathrm{Ct}$ values determined from the amplification curves of the samples are shown in Tables S1-3. The average $\mathrm{Ct}$ value ranged from 17 (for $C Y P 2$ ) to 25 (for $\mathrm{GzC} 2 \mathrm{HO} 44$ ). The variation in the expression of the candidate reference genes under the various conditions were assessed by BestKeeper, geNorm, and NormFinder.

BestKeeper analysis (http://www.gene-quantification.de/ bestkeeper.html): The BestKeeper tool expresses variation in gene expression as the standard deviation (SD) and coefficient of variance $(\mathrm{CV})$ of the derived $\mathrm{Ct}$ values from each reference candidate gene. Descriptive statistics of the candidate genes under four different culture conditions are provided in Table S4-9. A low SD should be expected for most stably expressed genes under the various conditions. The SD values of all of the genes (except $E F 1 B$ and $G A P D H$ ) were not greater than 1.0 (i.e., two-fold change) during the 6-day hyphal growth stage in $\mathrm{CM}$ liquid medium. The gene with the lowest expression levels under these conditions was $\mathrm{GzC} 2 \mathrm{HO} 44$ with an SD of 0.24, which represents an acceptable 1.18-fold change in expression. The expressions of $G z U B H$ and $G z F L O$ also varied similarly (Table 2). In the mycelia growth condition on carrot agar for 6 days, $E F 1 A$ was the most stable gene with an SD of 0.16, followed by GzRPS16, EF1B, GzSNC1, 
Table 2. Ranking of the candidate reference genes for normalization under mycelial growth in CM and AG by three statistical algorithms

\begin{tabular}{|c|c|c|c|c|c|c|c|c|c|c|c|c|}
\hline \multirow{3}{*}{ Rank } & \multicolumn{6}{|c|}{$\mathrm{CM}$} & \multicolumn{6}{|c|}{$\mathrm{AG}$} \\
\hline & \multicolumn{2}{|c|}{ BestKeeper } & \multicolumn{2}{|c|}{ geNorm } & \multicolumn{2}{|c|}{ NormFinder } & \multicolumn{2}{|c|}{ BestKeeper } & \multicolumn{2}{|c|}{ geNorm } & \multicolumn{2}{|c|}{ NormFinder } \\
\hline & Gene & $\mathrm{SD}[ \pm \mathrm{Ct}]$ & Gene & M value & Gene & Value & Gene & $\mathrm{SD}[ \pm \mathrm{Ct}$ & Gene & M value & Gene & Value \\
\hline 1 & $\mathrm{GzC} 2 \mathrm{H} 044$ & 0.24 & $G z U B H$ & 0.118 & $G z F L O$ & 0.011 & $G z R B P 1$ & 0.32 & GzRPS16 & 0.123 & $\mathrm{GzC} 2 \mathrm{HO} 44$ & 0.144 \\
\hline 2 & $G z U B H$ & 0.27 & $G z F L O$ & 0.133 & GzRPS16 & 0.013 & GzRPS16 & 0.33 & $G z R B P 1$ & 0.132 & EF1A & 0.196 \\
\hline 3 & GzFLO & 0.29 & $U B C$ & 0.138 & $G z R B P 1$ & 0.023 & $E F 1 A$ & 0.38 & $E F 1 A$ & 0.162 & CYP1 & 0.219 \\
\hline 4 & $G z S N C 1$ & 0.41 & $B T U B$ & 0.156 & $E F 1 A$ & 0.049 & $E F 1 B$ & 0.42 & $\mathrm{GzC} 2 \mathrm{H} 044$ & 0.188 & GzRPS16 & 0.230 \\
\hline 5 & GzRPS16 & 0.42 & $\mathrm{GzC} 2 \mathrm{HO} 044$ & 0.168 & $G z U B H$ & 0.070 & CYP2 & 0.43 & $G z S N C 1$ & 0.36 & $G z R B P 1$ & 0.272 \\
\hline 6 & $U B C$ & 0.45 & $G z R B P 1$ & 0.182 & $\mathrm{Gz} C 2 \mathrm{HO} 044$ & 0.078 & $B T U B$ & 0.45 & CYP2 & 0.361 & GzHSP70 & 0.281 \\
\hline 7 & $B T U B$ & 0.48 & GzRPS16 & 0.195 & $B T U B$ & 0.089 & $\mathrm{GzC} 2 \mathrm{H} 044$ & 0.47 & $E F 1 B$ & 0.412 & $U B C$ & 0.288 \\
\hline 8 & $G z D G A$ & 0.55 & $E F 1 A$ & 0.205 & CYP2 & 0.090 & $C Y P 1$ & 0.50 & $G z H S P 70$ & 0.419 & $G z U B H$ & 0.297 \\
\hline 9 & $G z R B P 1$ & 0.61 & $G z S N C 1$ & 0.227 & $U B C$ & 0.156 & GzSNC1 & 0.77 & $G z U B H$ & 0.443 & $B T U B$ & 0.354 \\
\hline 10 & GzHSP70 & 0.72 & $G z D G A$ & 0.24 & $G z S N C 1$ & 0.159 & GzHSP70 & 0.77 & $U B C$ & 0.456 & $G z S N C 1$ & 0.381 \\
\hline 11 & & 0.86 & GzHSP70 & 0.276 & $G z D G A$ & 0.203 & $U B C$ & 0.84 & $B T U B$ & 0.491 & $G z D G A$ & 0.437 \\
\hline 12 & $E F 1 A$ & 0.92 & $G A P D H$ & 0.309 & CYP1 & 0.255 & $G z U B H$ & 0.85 & $G z D G A$ & 0.55 & $E F 1 B$ & 0.542 \\
\hline 13 & $C Y P 1$ & 0.98 & $C Y P 2$ & 0.361 & $G A P D H$ & 0.300 & $G z D G A$ & 1.12 & $C Y P 1$ & 0.664 & $G z F L O$ & 0.561 \\
\hline 14 & $E F 1 B$ & 1.08 & $E F 1 B$ & 0.412 & GzHSP70 & 0.324 & GzFLO & 1.21 & $G z F L O$ & 0.729 & CYP2 & 0.594 \\
\hline \multirow[t]{2}{*}{15} & $G A P D H$ & 1.32 & CYP1 & 0.459 & $E F 1 B$ & 0.385 & $G A P D H$ & 1.33 & $G A P D H$ & 0.781 & $G A P D H$ & 0.616 \\
\hline & & & \multicolumn{2}{|c|}{$\begin{array}{l}G z F L O \& G z U B H^{\mathrm{a}} \\
(\mathrm{V} 2 / 3=0.047)\end{array}$} & $\begin{array}{l}\text { GzFLO \& } \\
\text { GzRPS16 }\end{array}$ & \multicolumn{2}{|l|}{0.009} & & \multicolumn{3}{|c|}{$\begin{array}{l}G z R B P 1 \& \text { \&zRPS16 } \sigma^{\mathrm{a}} E F 1 A \& \\
(\mathrm{~V} 2 / 3=0.074)\end{array}$} & 0.078 \\
\hline
\end{tabular}

${ }^{\mathrm{a}}$ Optimum pair of reference genes based on the average pairwise variation $\mathrm{V}$.

'Optimum pair of reference genes.

$G z U B H$, and $G z R B P 1$; the remaining genes (except $B T U B$ ) still expressed within a two-fold range $(\mathrm{SD}<1.0)$ (Table 3$)$. The variation in the expression of the genes under the perithecial induction stage on carrot agar was approximately two-fold higher than under mycelial growth conditions on carrot agar. The most stable gene under this condition was $G z R P S 16(\mathrm{SD}=0.46)$, followed by $E F 1 A$ and $G z F L O$. Four genes (GzDGA, GzC2H044, GAPDH, and $G z R B P 1$ ) displayed SD values greater than 1.0 (Table 3). When assessed over the entire sexual development stage on carrot agar, GzRPS16 was the most stable ( $\mathrm{SD}=0.37)$, followed by GzFLO and GzSNC1 (Table 3). Under trichothecene production conditions for 6 days, GzRBP1 was the most stable ( $\mathrm{SD}=0.32$ ). GzRPS16, EF1A, and GzRBP1 also showed SD values of less than 0.4 (Table 2). When all the conditions were combined, the most stable gene was $E F 1 A$ ( $\mathrm{SD}=0.64)$, although three other genes $(\mathrm{Gz} U B H$, $G z R B P 1$, and $U B C$ ) had similar SD levels (Table 4).

geNorm analysis: This algorithm defines the reference gene stability measure ( $\mathrm{M}$ value) by determining the pairwise variation $(\mathrm{V})$ in a reference gene compared to all other reference genes, and enables selection of the optimal reference genes for normalization. Genes with the lowest $\mathrm{M}$ values have the most stable expression. Ranking of candidate reference genes and the optimum pair of reference genes under each condition are shown in Tables $2-4$ and Table S10. Highly ranked genes displayed very high or high reference target stabilities (average $\mathrm{M} \leq 0.2$ and $\leq 0.5$, respectively), which is typically seen when reference targets are stably expressed or when evaluating candidate reference targets in a homogeneous set of samples (Hellemans et al., 2007). In CM, all genes had high expression stability with low M-values (below the default limit of $\mathrm{M}=0.5$ ) and the most stable gene was $G z U B H(\mathrm{M}=0.12)$, followed by $G z F L O$ and $U B C$. A low V value of 0.047 between $G z F L O$ and $G z U B H$, which is much lower than the common cut-off threshold of 0.15 , indicated that these genes can be used as references for normalization (Table 2). During the sexual developmental process on carrot agar, EF1A and GzRPS16, and $C Y P 1$ and $G z R P S 16$ were the most stable pairs over the 6-day vegetative growth and subsequent perithecial production stages, respectively. When the two stages were combined, GzRPS16 and EF1A were the best combination of reference genes (Table 3). Under trichothecene production conditions, GzRBPI and EF1A were highly stable (Table 2). Only three genes $(U B C, G z H S P 70$, and $G z U B H)$ displayed $\mathrm{M}$ values lower than 0.5 , all of which were identified as the best combination of reference genes under combined conditions (Table 4). For the combined data set, the pairwise variation in $U B C$ and GzHSP70 was higher than 0.15 , so a third gene (GzRPS16) was added to 
Table 3. Ranking of the candidate reference genes for normalization under the sexual development by three statistical algorithms

\begin{tabular}{|c|c|c|c|c|c|c|c|c|c|c|c|c|c|c|c|}
\hline \multirow{3}{*}{ Rank } & \multicolumn{5}{|c|}{ carrot-vegetative } & \multicolumn{5}{|c|}{ carrot-perithecial } & \multicolumn{5}{|c|}{ combined on carrot } \\
\hline & \multicolumn{2}{|c|}{ BestKeeper } & \multicolumn{2}{|c|}{ geNorm } & NormFinder & \multicolumn{2}{|c|}{ BestKeeper } & \multicolumn{2}{|c|}{ geNorm } & NormFinder & \multicolumn{2}{|c|}{ BestKeeper } & geNorm & \multicolumn{2}{|c|}{ NormFinder } \\
\hline & Gene & $\begin{array}{c}\mathrm{SD} \\
{[ \pm \mathrm{Ct}]}\end{array}$ & Gene & $\begin{array}{c}\mathrm{M} \\
\text { value }\end{array}$ & Gene & Value Gene & $\begin{array}{r}\mathrm{SD} \\
{[ \pm \mathrm{Ct}]}\end{array}$ & Gene & $\begin{array}{c}\mathrm{M} \\
\text { value }\end{array}$ & Gene & Value Gene & $\begin{array}{c}\mathrm{SD} \\
{[ \pm \mathrm{Ct}]}\end{array}$ & Gene & $\begin{array}{c}\mathrm{M} \\
\text { value }\end{array}$ & Value \\
\hline 1 & $E F 1 A$ & 0.16 & $E F 1 A$ & 0.118 & GzSNC1 & $0.113 B T U B$ & 0.40 & GzRPS16 & .168 & $U B C$ & $0.147 \mathrm{GzRPS16}$ & 0.37 & GzRPS16 & $0.367 U B C$ & 0.182 \\
\hline 2 & GzRPS16 & 0.20 & GzRPS16 & 0.126 & EFIA & 0.149 GzRPS16 & 0.46 & CYP1 & 0.197 & $G z U B H$ & $0.202 \mathrm{GzFLO}$ & 0.44 & EF1A & 0.377 EFlA & 0.256 \\
\hline 3 & $E F 1 B$ & 0.21 & $G z U B H$ & 0.132 & GzRPS16 & $0.182 \mathrm{EF} 1 \mathrm{~A}$ & 0.48 & EFIA & 0.213 & $E F 1 B$ & $0.242 \mathrm{GzSNC1}$ & 0.49 & $U B C$ & $0.385 \mathrm{Gz} U B H$ & 0.288 \\
\hline 4 & $G z S N C 1$ & 0.24 & GzSNC1 & 0.179 & $U B C$ & $0.206 \mathrm{GzFLO}$ & 0.49 & $U B C$ & 0.311 & EF1A & $0.261 \mathrm{Gz} U B H$ & 0.57 & $E F 1 B$ & 0.427 GzRPS16 & 0.299 \\
\hline 5 & $G z U B H$ & 0.30 & $G z R B P 1$ & 0.212 & $G z R B P 1$ & $0.224 C Y P 1$ & 0.55 & $E F 1 B$ & 0.358 & $G z S N C 1$ & $0.265 E F 1 A$ & 0.58 & CYP2 & $0.443 G z S N C 1$ & 0.314 \\
\hline 6 & $G z R B P 1$ & 0.30 & CYP2 & 0.241 & $G z U B H$ & $0.229 E F 1 B$ & 0.64 & $G z D G A$ & 0.367 & CYP2 & $0.322 C Y P 1$ & 0.59 & $G z U B H$ & $0.479 G z D G A$ & 0.374 \\
\hline 7 & $\mathrm{Gz} C 2 \mathrm{HO} 44$ & 0.38 & $U B C$ & 0.276 & $G z F L O$ & $0.251 C Y P 2$ & 0.64 & CYP2 & 0 & $A P D H$ & $0.339 U B C$ & 0.65 & $C Y P 1$ & 0.508 CYP2 & .396 \\
\hline 8 & GzFLO & 0.38 & $E F 1 B$ & 0.3 & $E F 1 B$ & $0.283 \mathrm{GzSNC1}$ & 0.75 & $G z U B H$ & 5 & GzRPS16 & $0.380 \mathrm{GzDGA}$ & 0.71 & GzFLO & $0.533 E F 1 B$ & .397 \\
\hline 9 & CYP2 & 0.43 & GzFLO & 0.335 & CYP2 & 0.294 GzHSP70 & 0.75 & GzFLO & 0.471 & CYP1 & $0.388 \mathrm{GzHSP} 70$ & 0.71 & $G z D G A$ & $0.552 \mathrm{GzHSP} 70$ & 0.420 \\
\hline 10 & $G z D G A$ & 0.50 & $G z D G A$ & 0.367 & $G z D G A$ & $0.310 U B C$ & 0.76 & $G z S N C 1$ & 0.514 & GzHSP70 & 0.400 CYP2 & 0.71 & GzHSP70 & $0.58 \mathrm{GzFLO}$ & 0.429 \\
\hline 11 & $U B C$ & 0.52 & GAPDH & 0.409 & $G A P D H$ & $0.398 \mathrm{Gz} U B H$ & 0.83 & GzHSP70 & 0.549 & $G z D G A$ & $0.431 E F 1 B$ & 0.76 & GzSNC1 & $0.605 G z R B P 1$ & 0.436 \\
\hline 12 & GzHSP70 & 0.53 & CYP1 & 0.439 & $\mathrm{Gz} C 2 \mathrm{H} 044$ & $0.401 G z D G A$ & 1.00 & $G A P D H$ & 0.621 & $B T U B$ & $0.528 G z R B P 1$ & 0.77 & $G z R B P 1$ & $0.649 C Y P 1$ & 0.445 \\
\hline 13 & GAPDH & 0.57 & GzHSP70 & 0.47 & GzHSP70 & $0.403 \mathrm{GzC} 2 \mathrm{H} 044$ & 1.03 & $B T U B$ & 0.661 & GzFLO & $0.552 B T U B$ & 0.80 & $\mathrm{Gz} C 2 \mathrm{H} 044$ & $0.693 G A P D H$ & 0.484 \\
\hline 14 & CYP1 & 0.66 & $\mathrm{GzC} 2 \mathrm{HO} 44$ & 0.503 & $C Y P 1$ & $0.466 G A P D H$ & 1.04 & $G z R B P 1$ & 0.706 & $G z R B P 1$ & $0.566 \mathrm{GzC} 2 \mathrm{H} 044$ & 0.87 & $G A P D H$ & $0.731 \mathrm{GzC} 2 \mathrm{H} 044$ & 0.496 \\
\hline \multirow[t]{2}{*}{15} & $B T U B$ & 1.04 & $B T U B$ & 0.668 & BTUB & $0.803 \mathrm{GzRBP1}$ & 1.26 & $\mathrm{GzC} 2 \mathrm{H} 044$ & 0.758 & $\mathrm{GzC} 2 \mathrm{HO} 44$ & $0.594 G A P D H$ & 1.07 & $B T U B$ & $0.771 B T U B$ & 0.745 \\
\hline & & & $\begin{array}{l}\text { GzRPS16 } \\
\text { (V2/3=0.0 }\end{array}$ & $F 1$ & $\begin{array}{l}{ }^{\mathrm{a}} G z S N C 1 \& \\
E F 1 A^{\mathrm{b}}\end{array}$ & 0.072 & & $\begin{array}{l}C Y P 1 \& G z \\
(\mathrm{~V} 2 / 3=0.08\end{array}$ & $\operatorname{PS} 16^{\mathrm{a}}$ & $\begin{array}{l}U B C \& \\
G z U B H^{\mathrm{b}}\end{array}$ & 0.104 & & $\begin{array}{l}E F 1 A \& G z I \\
(\mathrm{~V} 2 / 3=0.08)\end{array}$ & $\begin{array}{r}P S 16^{\mathrm{a}} E F 1 A \& \\
G z U B H^{\mathrm{b}}\end{array}$ & 0.148 \\
\hline
\end{tabular}

${ }^{\mathrm{a}}$ Optimum pair of reference genes based on the average pairwise variation $\mathrm{V}$.

'Optimum pair of reference genes. 
Table 4. Ranking of the candidate reference genes for normalization across all conditions by three statistical algorithms

\begin{tabular}{|c|c|c|c|c|c|c|}
\hline \multirow{2}{*}{ Rank } & \multicolumn{2}{|c|}{ "BestKeeper } & \multicolumn{2}{|c|}{ geNorm } & \multicolumn{2}{|c|}{ NormFinder } \\
\hline & Gene & $\mathrm{SD}[ \pm \mathrm{Ct}]$ & Gene & M value & Gene & Value \\
\hline 1 & $E F 1 A$ & 0.65 & $U B C$ & 0.409 & $G z U B H$ & 0.213 \\
\hline 2 & $G z U B H$ & 0.66 & GzHSP70 & 0.445 & $E F 1 A$ & 0.219 \\
\hline 3 & $G z R B P 1$ & 0.66 & $G z U B H$ & 0.467 & CYP1 & 0.286 \\
\hline 4 & $U B C$ & 0.69 & $G z R B P 1$ & 0.557 & GzRPS16 & 0.327 \\
\hline 5 & $G z S N C 1$ & 0.70 & $E F 1 A$ & 0.606 & $G z R B P 1$ & 0.335 \\
\hline 6 & GzRPS16 & 0.70 & CYP1 & 0.67 & GzHSP70 & 0.353 \\
\hline 7 & $\mathrm{GzC} 2 \mathrm{H} 044$ & 0.75 & $G z D G A$ & 0.704 & $U B C$ & 0.419 \\
\hline 8 & GzHSP70 & 0.75 & GzRPS16 & 0.726 & $G z D G A$ & 0.424 \\
\hline 9 & $B T U B$ & 0.77 & GzSNC1 & 0.745 & $E F 1 B$ & 0.429 \\
\hline 10 & $G z F L O$ & 0.78 & $C Y P 2$ & 0.773 & $\mathrm{GzC} 2 \mathrm{H} 044$ & 0.454 \\
\hline 11 & $E F 1 B$ & 0.80 & $E F 1 B$ & 0.793 & CYP2 & 0.492 \\
\hline 12 & CYP1 & 0.81 & $G z F L O$ & 0.817 & $G z S N C 1$ & 0.494 \\
\hline 13 & $C Y P 2$ & 0.89 & $\mathrm{GzC} 2 \mathrm{H} 044$ & 0.843 & $G z F L O$ & 0.495 \\
\hline 14 & $G z D G A$ & 0.90 & $G A P D H$ & 0.932 & $B T U B$ & 0.546 \\
\hline \multirow[t]{2}{*}{15} & $G A P D H$ & 1.51 & $B T U B$ & 1.023 & $G A P D H$ & 0.936 \\
\hline & \multicolumn{4}{|c|}{ GzRPS16, GzHSP70, \& UBC $(\mathrm{V} 3 / 4=0.147)$} & $G z U B H \& E F 1 A^{\mathrm{b}}$ & 0.106 \\
\hline
\end{tabular}

${ }^{a}$ Optimum pair of reference genes based on the average pairwise variation $\mathrm{V}$.

${ }^{\mathrm{b}}$ Optimum pair of reference genes.

normalize gene expression (Table 4).

NormFinder analysis. Unlike the geNorm algorithm, NormFinder identifies the optimal reference genes among a set of candidate genes based on the stability of gene expression between the groups, resulting in an optimum pair of reference genes (Table S11). Under CM conditions, eight genes had expression stability values of less than 0.1 . The most stable gene was $G z F L O$, followed by GzRPS16 and $G z R B P 1$. When two genes are used as references for normalization, GzFLO and GzRPS16 would be the best combination because they had stability values $(0.009)$ less than that of the single most stable gene ( $G z F L O$, stability value of 0.011) (Table 2). During vegetative growth on carrot agar, four genes showed stability values less than 0.2 . The three most stable genes were GzSNC1, EF1A, and GzRPS16; GzSNC1 and EF1A were the best combination. During the perithecial induction stage, only $U B C$ had a stability value of less than 0.2 , followed by $G z U B H$; these genes achieved values of 0.104 (Table 3). For the combined data sets for sexual development, $U B C$ was ranked as the most stably expressed gene $(>0.2)$, followed by $E F 1 A$ and $G z U B H$, but the combination of second- and third-ranked genes $(E F 1 A$ and $G z U B H)$ achieved a lower stability value than that of $U B C$ (Table 3). Under AG conditions, $\mathrm{GzC} 2 \mathrm{HO} 044$ and $E F 1 A$ were ranked as the best genes, but $E F 1 A$ and the eight-ranked $\mathrm{Gz} U B H$ gene ( $\mathrm{SV}=0.297$ ) were identified as the optimum reference pair (Table 2). When all conditions were combined, the reference gene rank was: $G z U B H>$ EF1A > CYP1 (Table 4).

Identification of best candidate reference genes by combining three programs. The output data from geNorm and NormFinder can be easily compared because they both use the same input data (linear expression values), whereas that of BestKeeper may be different as it uses $\mathrm{Ct}$ values. Nevertheless, it was possible to select the best reference genes from the top three most stable genes identified by each analysis. In CM, GzFLO was always identified as one of the best reference genes (third in BestKeeper, second in geNorm, and first in NormFinder). As an alternative, $G z U B H$ ranked second in BestKeeper, first in geNorm, and fourth in NormFinder. Similarly, both EF1A and GzRPS16 were stably expressed during both vegetative and perithecial induction conditions on carrot agar. These two genes were also identified as the best reference genes under the AG condition. When all conditions were combined, $G z U B H$ (second in BestKeeper, third in geNorm, and first in NormFinder), and EFIA (first in BestKeeper, fifth in geNorm, and second in NormFinder) were very stable. The primers for $G z U B H, G z R P S 16$, and $G z F L O$ have been submitted to the RTPrimerDB (with ID 8322, 8323, and 8324 , respectively). GAPDH was among the least stably expressed genes under most conditions. 


\section{Discussion}

This study evaluated numerous culture conditions, candidate genes, and algorithms to select the best reference genes for qRT-PCR, of which little is known in filamentous fungi. The conditions tested here can be divided into different categories based on fungal developmental or physiological status. The conditions in $\mathrm{CM}$ and $\mathrm{AG}$, and on carrot agar favor only mycelial growth in F. graminearum; none of the other developmental processes such as asexual sporulation, sexual fruiting body formation, and sexual sporulation could be stimulated. However, the physiological or metabolic status is diverse among these conditions. In CM, metabolic pathways associated with high nutrient use are activated, whereas on carrot agar those for adapting to nitrogen starvation are activated, which is considered a prerequisite for switching to the sexual reproductive mode. The AG liquid culture specifically stimulates pathways producing several secondary metabolites, such as trichothecenes (Gardiner et al., 2009) In contrast, perithecial formation on carrot agar is heterogeneous in terms of fungal development. Even though this represents a typical sexual development process in $F$. graminearum, it also includes asexual spores as well as ground mycelia. In addition to the culture conditions, we assessed the time-course variation in gene expression by monitoring two or three time points under each condition. Because these diverse conditions represent primary developmental and/or physiological stages in F. graminearum, this increases the reliability of the reference genes selected in this study.

The candidate reference genes evaluated here are more diverse in terms of number and class than those in previous reports. Seven housekeeping genes, which have been commonly used as endogenous controls in various organisms, were assessed simultaneously under the various conditions using three algorithms. In most $F$. graminearum studies, only single housekeeping gene, such as actin, $B T U B, E F 1 A$, $E F 1 B$, or $18 \mathrm{~S}$ rRNA, is used as an internal control without in-depth evaluation (Chen et al., 2011; Gardiner et al., 2009; Lee et al., 2009; Liu et al., 2011; Pandolfi et al., 2010). Lysøe et al. (Lysøe et al., 2009) evaluated three housekeeping genes (BTUB, EFIA, and UBC) using BestKeeper, but did not provide detailed data.

The results of the present study question the reliability of housekeeping genes as internal controls in qRT-PCR analysis. We found that $G A P D H$ is an unsuitable reference gene, as previously demonstrated in animals (Barber et al., 2005; Olsvik et al., 2005). In addition, other housekeeping genes such as $E F 1 B, C Y P 1$, and $C Y P 2$ were ranked among the least stable genes under certain growth conditions. Only two housekeeping genes (EF1A and UBC) displayed relatively stable expression patterns under all conditions.
Among the eight new candidate genes, three had expression stabilities similar to the housekeeping genes. Interestingly, two of the three genes exhibited the features similar to commonly used housekeeping genes, supporting the reliability of the procedure for selecting new reference genes using RNA-seq data. $G z U B H$, annotated as a hypothetical protein carrying an ubiquitin carboxyl-terminal hydrolase domain, may be involved in deubiquitination (the process of removing ubiquitin molecules from polyubiquitinated peptides) in F. graminearum. Because the $U B C$ gene encoding ubiquitin-conjugating enzyme is frequently used as reference gene in various organisms including $F$. graminearum, it is possible that other genes, such as $G z U B H$, which is involved in the ubiquitin-mediated protein degradation pathway can be stably expressed under several conditions in F. graminearum and other organisms. GzRPS16 has been annotated as a mitochondrial ribosomal protein S16 that characterized as a human housekeeping gene (Eisenberg and Levanon, 2003). In addition, ribosomal protein S4 has been used as a reference gene for qRTPCR in a mycorrhizal fungus (Govindarajulu et al., 2005). RPS3 and RPS18 were the most stable in a fungus-infected red flour beetle (Lord et al., 2010). In contrast to these genes, $G z F L O$, annotated as a hypothetical protein similar to the putative cell surface flocculin, would be a good reference gene specific to only the $\mathrm{CM}$ condition because it was not stable under the other conditions.

The present study suggests that these genes can function as reference genes in other filamentous fungi as well as in $F$. graminearum. In addition, these genes can be applied to other conditions not examined in this study because the three conditions tested here represent the primary physiological processes in F. graminearum. However, evaluation of these genes in planta is required.

\section{Acknowledgments}

This study was carried out with the support of "Cooperative Research Program for Agricultural Science \& Technology Development (Project No. PJ007340)", and supported by a grant from the Next-Generation BioGreen 21 Program (No. PJ008210), Rural Development Administration, Republic of Korea.

\section{Supplementary data}

Supplementary data (Tables S1-11) associated with this article can be found.

\section{References}

Anderson, C. L., Ledet-Jensen, J. and Ørntoft, T. 2004. Normal- 
ization of real-time quantitative RT-PCR data: a model-based variance estimation approach to identify genes suited for normalization-applied to bladder and colon cancer datasets. Cancer Res. 64:5245-5250.

Barber, R. D., Harmer, D. W., Coleman, R. A. and Clark, B. J. 2005. GAPDH as a housekeeping gene: analysis of GAPDH mRNA expression in a panel of 72 human tissues. Physiol. Genomics 21:389-395.

Bustin, S. A. and Nolan, T. 2004. Pitfalls of quantitative real-time reverse transcription polymerase chain reaction. J. Biomol. Tech. 15:155-166.

Chen, F., Zhang, J., Song, X., Yang, J., Li, H., Tang, H., Liao, and Y.-C. 2011. Combined metabonomic and quantitative realtime PCR analyses reveal systems metabolic changes of Fusarium graminearum induced by Tri5 gene deletion. J. Proteome Res. 10:2273-2285.

Cuomo, C. A., Güldener, U., Xu, J.-R., Trail, F. et al. 2007. The Fusarium graminearum genome reveals a link between localized polymorphism and pathogen specialization. Science 317:1400-1402.

Czechowski, T., Stitt, M., Altmann, T., Udvardi, M. K. and Scheible, W. R. 2005. Genome-wide identification and testing of superior reference genes for transcript normalization in Arabidopsis. Plant Physiol. 139:5-17.

Desjardins, A. E. and Proctor, R. H. 2007. Molecular biology of Fusarium mycotoxins. Int. J. Food Microbiol. 119:47-50.

Dheda, K., Huggett, J. F., Bustin, S. A., Johnson, M. A., Rook, G. and Zumla, A. 2004. Validation of housekeeping genes for normalizing RNA expression in real-time PCR. Biotechniques. 37:112-119.

Eisenberg, E. and Levanon, E. Y. 2003. Human housekeeping genes are compact. Trends Genet. 19:362-365.

Gardiner, D. M., Kazan, K. and Manners, J. M. 2009. Novel genes of Fusarium graminearum that negatively regulate deoxynivalenol production and virulence. Mol. Plant-Microbe Interact. 12:1588-1600.

Gardiner, D. M., Kazan, K. and Manners, J. M. 2009. Nutrient profiling reveals potent inducers of trichothecene biosynthesis in Fusarium graminearum. Fungal Genet. Biol. 46:604-613.

Govindarajulu, M., Pfeffer, P. E., Jin, H., Abubaker, J., Douds, D. D., Allen, J. W., Bucking, H., Lammers, P. J. and Shachar-Hill, Y. 2005. Nitrogen transfer in the arbuscular mycorrhizal symbiosis. Nature 435:819-823.

Güldener, U., Seong, K. Y., Boddu, J., Cho, S., Trail, F., Xu, J.-R., Adam, G., Mewes, H. W., Muehlbauer, G. J. and Kistler, H. C. 2006. Development of a Fusarium graminearum Affymetrix GeneChip for profiling fungal gene expression in vitro and in planta. Fungal Genet. Biol. 43:316-325.

Gutierrez, L., Moritz, M., Guénin, S., Pelloux, J., Lefebvre, J. F., Louvet, R., Rusterucci, C., Moritz, T., Guerineau, F., Bellini, C. and Van Wuytswinkel, O. 2008. The lack of a systematic validation of reference genes: a serious pitfall undervalued in reverse transcription-polymerase chain reaction (RT-PCR) analysis in plants. Plant Biotechnol. J. 6:609-618.

Hallen, H. E., Huebner, M., Shiu, S. H., Güldener, U. and Trail, F. 2007. Gene expression shifts during perithecium development in Gibberella zeae (anamorph Fusarium graminearum), with particular emphasis on ion transport proteins. Fungal Genet. Biol. 44 :1146-1156.

Hellemans, J., Mortier, G., De Paepe, A., Speleman, F. and Vandesompele, J. 2007. qBase relative quantification framework and software for management and automated analysis of real-time quantitative PCR data. Genome Biol. 8:R19.

Lee, P. D., Sladek, R., Greenwood, C. M. and Hudson, T. J. 2002. Control genes and variability: absence of ubiquitous reference gene transcripts in diverse mammalian expression studies. Genome Res. 12:292-297.

Lee, S.-H., Lee, J., Lee, S., Park, E.-H., Kim, K.-W., Kim, M.-D., Yun, S.-H. and Lee, Y.-W. 2009. GzSNF1 is required for normal sexual and asexual development in the ascomycete Gibberella zeae. Eukaryot. Cell 8:116-127.

Lee, S.-H., Lee, S., Choi, D., Lee, Y.-W. and Yun, S.-H. 2006. Identification of the down-regulated genes in a mat1-2-deleted strain of Gibberella zeae, using cDNA subtraction and microarray analysis. Fungal Genet. Biol. 43:295-310.

Leslie, J. F. and Summerell, B. A. 2006. The Fusarium lab manual, Blackwell, Ames,

Liu, X., Fu, J., Yun, Y., Yin, Y. and Ma, Z. 2011. A sterol C-14 reductase encoded by $F g E R G 24 B$ is responsible for the intrinsic resistance of Fusarium graminearum to amine fungicides. Microbiology 157:1665-1675.

Lord, J. C., Hartzer, K., Toutges, M. and Oppert, B. 2010. Evaluation of quantitative PCR reference genes for gene expression studies in Tribolium castaneum after fungal challenge. $J$. Microbiol. Methods 80:219-221.

Lysøe, E., Bone, K. R. and Klemsdal, S. S. 2009. Real-time quantitative expression studies of the zearalenone biosynthetic gene cluster in Fusarium graminearum. Phytopahtology 99:176-184.

McMullen, M., Jones, R. and Gallenberg, D. 1997. Scab of wheat and barley: a re-emerging disease of devastating impact. Plant Dis. 81:1340-1348.

O'Donnell, K., Kistler, H. C., Tacke, B. K. and Casper, H. H. 2000. Gene genealogies reveal global phylogeographic structure and reproductive isolation among lineages of Fusarium graminearum, the fungus causing wheat scab. Proc. Natl. Acad. Sci. USA 97:7905-7910.

Olsvik, P. A., Lie, K. K., Jordal, A. O., Nilsen, T. O. and Hordvik, I. 2005. Evaluation of potential reference genes in real-time RT-PCR studies of Atlantic salmon. BMC Mol. Biol. 6:21.

Pandolfi, V., Jorge, E. C., Melo, C. M. R., Albuquerque, A. C. S. and Carrer, H. 2010. Gene expression profile of the plant pathogen Fusarium graminearum under the antagonistic effect of Pantoea agglomerans. Genet. Mol. Res. 9:12981311.

Pfaffl, M. W. 2001. A new mathematical model for relative quantification in real-time RT-PCR. Nucleic Acids Res. 29:20022007.

Pfaffl, M. W., Tichopad, A., Prgomet, C. and Neuvians, T. P. 2004. Determination of stable housekeeping genes, differentially regulated target genes and sample integrity: BestKeeperExcel-based tool using pair-wise correlations. Biotechnol. 
Lett. 26:509-515.

Seong, K. Y., Zhao, X., Xu, J. R., Güldener, U. and Kistler, H. C. 2008. Conidial germination in the filamentous fungus Fusarium graminearum. Fungal Genet. Biol. 45:389-399.

Stephens, A. E., Gardiner, D. M., White, R. G., Munn, A. L. and Manners, J. M. 2008. Phases of Fusarium graminearum development and gene expression during crown rot disease of wheat. Mol. Plant-Microbe Interact. 21:1571-1581.

Suzuki, T., Higgins, P. J. and Crawford, D. R. 2000. Control selection for RNA quantitation. Biotechniques 29:332-337.

Vandesompele, J., De Preter, K., Pattyn, F., Poppe, B., Van Roy, N., De Pape, A. and Speleman, F. 2002. Accurate normalization of real-time quantitative PCR data by genometric averaging of multiple internal control genes. Genome Biol. 3:e34. 